\title{
COMPACTNESS AND ORDINALS
}

\author{
by C. J. KNIGHT
}

(Received 20 November, 1970; revised 29 March, 1971)

If $p$ and $q$ are cardinal numbers and $E$ is a topological space, then the following property may or may not hold:

Every cover of $E$ by fewer than $q$ open sets has a subcover by fewer than $p$ of them. (1) Clearly these properties, for various numbers $p$ and $q$, are far from being independent; in this paper, we investigate some of the interrelationships between them.

Properties like (1) were originally considered by Alexandroff and Urysohn in [1]. An account of some of them is given by I. S. Gál in [2], [3] and [4]. Gál defines a space to be $(m, n)$-compact if every cover by at most $n$ open sets has a subcover by at most $m$ of them. We consider also the properties excluded by Gál's definition; that is, in (1) we allow $p$ or $q$ to be of the form $\aleph_{\lambda}$, where $\lambda$ is a limit ordinal. Although, as we shall see, the possession or otherwise of these properties for $p=\aleph_{\lambda}$ is determined by what happens for $p<\aleph_{\lambda}$, we may obtain extra information about the space $E$ by allowing $q$ to be of the form $\aleph_{\lambda^{\prime}}$.

We assume the axiom of choice, so that every infinite cardinal number is an aleph. For typographical reasons, we shall write $\aleph_{\alpha} \alpha$ instead of the customary $\aleph_{\alpha}$.

Defintion 1 . A topological space $E$ will be said to be $[\beta, \alpha]$-compact if and only if every cover of $E$ by fewer than $\aleph \alpha$ open sets has a subcover by fewer than $\aleph \beta$ of them.

Then Gál's $\left(\aleph_{\beta}, \aleph_{\alpha}\right)$-compactness is our $[\beta+1, \alpha+1]$-compactness. Clearly, these properties, for various $\alpha$ and $\beta$, are not independent: for, given the space $E$, Definition 1 establishes a relation $R$ on the ordinal numbers, according to which $\alpha R \beta$ if and only if $E$ is $[\beta, \alpha]$-compact; and this relation is evidently reflexive and transitive, and has the property that

$$
\text { if } \gamma \leqq \alpha \text { and } \beta \leqq \delta \text { and } \alpha R \beta \text {, then } \gamma R \delta \text {. }
$$

DEFINITION 2. If $E$ is a topological space and $\alpha$ is an ordinal number, then we shall write

$$
\mu_{E} \alpha=\min \{\beta: E \text { is }[\beta, \alpha] \text {-compact }\} \text {. }
$$

We shall abbreviate $\mu_{E}$ to $\mu$ when only one space is in question.

It is easy to see that the ordinal function $\mu$ contains all the information about $E$ given by the relation $R$ defined above; for from (2) there follows at once

THEOREM 1. $E$ is $[\beta, \alpha]$-compact if and only if $\mu \alpha \leqq \beta$.

The problem that we begin to study in this paper may now be stated as: Characterise functions on the ordinals that arise as $\mu_{E}$ for some topological space $E$.

THEOREM 2. The function $\mu$ is (non-strictly) increasing, and $\mu 0=0$.

Proof. Suppose that $\gamma \leqq \alpha$. Then (2) implies that $\gamma R \mu \alpha$, and Theorem 1 then says that $\mu \nu \leqq \mu \alpha$. That $\mu 0=0$ is immediate from the definitions. 
THEOREM 3. The function $\mu$ is idempotent: $\mu^{2}=\mu$.

Proof. As $\alpha R \alpha$, we know that $\mu \alpha \leqq \alpha$ and hence, by Theorem $2, \mu^{2} \alpha \leqq \mu \alpha$. However $\mu \alpha R \mu(\mu \alpha)$ and $\alpha R \mu \alpha$; so, as $R$ is transitive, $\alpha R \mu^{2} \alpha$, whence $\mu \alpha \leqq \mu^{2} \alpha$ by Theorem 1 . Thus $\mu^{2} \alpha=\mu \alpha$ for all $\alpha$.

The property of transitivity may be expressed in terms of $\mu$ as follows.

THEOREM 4. For each $\alpha, \mu(\alpha+1)$ is equal either to $\alpha+1$ or to $\mu \alpha$.

Proof. Clearly $\mu \alpha \leqq \mu(\alpha+1) \leqq \alpha+1$. Suppose that $\mu(\alpha+1)<\alpha+1$. Then $\mu(\alpha+1) \leqq \alpha$, and so $\mu(\alpha+1)=\mu^{2}(\alpha+1) \leqq \mu \alpha \leqq \mu(\alpha+1)$. Thus, if $\mu(\alpha+1) \neq \alpha+1$, then $\mu(\alpha+1)=\mu \alpha$.

Theorem 4 tells us what happens at successor-ordinals. The behaviour of $\mu$ at limitordinals is given by

THEOREM 5. The function $\mu$ is continuous.

Proof. Suppose that $\lambda$ is a limit-ordinal. Then every cover of $E$ by fewer than $N \lambda$ open sets comprises in fact fewer than $\aleph \alpha$ open sets for some $\alpha<\lambda$, and so has a subcover by fewer than $\aleph \mu \alpha$ of them. Thus $\mu \lambda \leqq \lim \mu \alpha$, and the reverse inequality holds since $\mu$ is an increasing function.

So far, $\mu$ has been regarded as a function defined on the class of all ordinals; but in fact it is determined by its values on a set of ordinals, for we have the following theorem.

THEOREM 6. The function $\mu$ is eventually constant.

Proof. Every open cover of $E$ has a subcover of cardinality at most that of $E$ itself.

If $f$ is any ordinal function, we shall write $f \infty=\kappa$ to mean that $f$ is eventually constant, with $f \alpha=\kappa$ for all large enough $\alpha$.

The description of $\mu_{E}$ given by Theorems 2 to 6 is complete provided that $E$ is of cardinality less than $\aleph \omega_{0}$. We shall prove

THEOREM 7. If $f$ is a continuous increasing ordinal function with $f 0=0$, and, for each $\alpha, f(\alpha+1)=$ either $\alpha+1$ or $f \alpha$, and with $f \infty<\omega_{0}$, then there is a completely regular space $E$ with $f=\mu_{\mathrm{E}}$.

The proof of this theorem is given after that of Theorem 9.

The space $E$ will be constructed as a discrete union of spaces of ordinals. We observe first that the class of all $\beta$ with $f(\beta+1)=\beta+1$ is finite; for each such $\beta$ is at most equal to $f \infty$, which is finite. Moreover, each $\beta$ is finite, and hence $\omega_{\beta}$ is regular. $E$ will be the discrete union of the spaces $\left\{\gamma: \gamma<\omega_{\beta}\right\}$, one for each $\beta$ with $f(\beta+1)=\beta+1$. To calculate $\mu_{E}$ we shall use

THEOREM 8. If $E$ is the discrete union of its subspaces $E_{i}(1 \leqq i \leqq k)$, then

$$
\mu_{E} \alpha=\max _{1 \leqq i \leqq k} \mu_{E_{i}} \alpha \text { for all } \alpha \text {. }
$$

Proof. Let $\Phi$ be a covering of $E$ by fewer than $\kappa \alpha$ open sets; then, for each $i$, there is a subclass $\Psi_{i}$ of $\Phi$ covering $E_{i}$ and of cardinality less than $\aleph \mu_{E_{i}} \alpha$. Then $\bigcup\left\{\Psi_{l}: 1 \leqq i \leqq k\right\}$ is a 
cover of $E$ of cardinality at most the sum of the cardinalities of the $\Psi_{i}$. Since there are only finitely many summands, this is less than $\aleph \max \mu_{E_{i}} \alpha$. Thus $\mu_{E} \alpha \leqq \max _{t} \mu_{E_{i}} \alpha$. On the other hand, suppose that $\max \mu_{E_{1}} \alpha$ is attained for $i=j$. Then, for each $\gamma$ less than $\mu_{E_{j}} \alpha$, there is a cover of $E_{j}$ by fewer than $\kappa \alpha$ open sets which has no subcover by fewer than $\kappa \gamma$ of them. This cover, together with $\left\{E_{i}: i \neq j\right\}$, makes a cover of $E$ by fewer than $\aleph \alpha$ open sets with no subcover by fewer than $\kappa \gamma$ of them. Thus $\max _{i} \mu_{E_{i}} \alpha=\mu_{E_{j}} \alpha \leqq \mu_{E} \alpha$, and so Theorem 8 is proved.

THEOREM 9. If $E$ is the space of all ordinal numbers less than $\omega_{\beta}$, with order-topology, where $\omega_{\beta}$ is regular, then $\mu_{E}$ is given by

$$
\mu_{E} \alpha=0 \text { for } \alpha \leqq \beta \text {, and } \mu_{E} \alpha=\beta+1 \text { for } \beta+1 \leqq \alpha .
$$

Proof. Since $\omega_{\beta}$ is regular, every increasing sequence of type less than $\omega_{\beta}$ consisting of ordinals less than $\omega_{\beta}$ has limit less than $\omega_{\beta}$.

Consider any infinite cover $\Phi$ of $E$ by $\mathcal{N} \phi$ open sets, with $\phi<\beta$, and suppose that $\Phi$ has no subcover of cardinality smaller than $\aleph \phi$. Let $\Phi$ be well-ordered as a sequence $\left\{U_{\gamma}: \gamma<\omega_{\phi}\right\}$. Then, for each $\delta<\omega_{\phi}$, the subset $\Phi_{\delta}$ of $\Phi$ defined by

$$
\Phi_{\delta}=\left\{U_{\gamma}: \gamma<\delta\right\}
$$

fails to cover $E$, for its cardinality is less than $\aleph \phi$. Let $\xi_{\delta}$ be the least member of $E \backslash \bigcup \Phi_{\delta}$. Then $\left(\xi_{\delta}: \delta<\omega_{\phi}\right)$ is an increasing sequence of type $\omega_{\phi}$ (which is less than $\omega_{\beta}$ ), consisting of ordinals in $E$, each of which is thus less than $\omega_{\beta}$, and so $\left(\xi_{\delta}\right)$ has a limit, $\xi$ say, less than $\omega_{\beta}$. That is, $\xi \in E$. But now $\xi$ belongs to some member $U_{\gamma}$ of $\Phi$, and thus, since $\omega_{\phi}$ is a limit ordinal, so do all the $\xi_{\delta}$ from some $\delta$ onwards. In particular, there is a $\delta$, greater than $\gamma$, with $\xi_{\delta} \in U_{\gamma}$. However, we then have $U_{\gamma} \in \Phi_{\delta}$, so that $\xi_{\delta} \in U \Phi_{\delta}$, which contradicts the definition of $\xi_{8}$.

Thus the supposition that $\Phi$ had no subcover by fewer than $\aleph \phi$ of its members was false, and so every infinite cover $\Phi$ of $E$ by fewer than $\aleph \beta$ open sets has a subcover of cardinality smaller than that of $\Phi$. If the subcover is still infinite, the argument can be repeated, yielding a strictly decreasing sequence of cardinalities. Since the cardinal numbers are well-ordered, every descending sequence of them is finite (see [5], p. 259, Theorem 1). Thus our process must terminate, necessarily with a subcover of $\Phi$ which is no longer infinite. So every cover of $E$ by fewer than $\aleph \beta$ open sets has finite subcover. That is, $\mu \beta=0$, from which it follows that $\mu \alpha=0$ for all $\alpha \leqq \beta$.

Finally, the cover of $E$ by all open initial segments $\{\alpha: \alpha<\gamma\}$, which is of cardinality $\aleph \beta$, has no subcover by fewer than $\aleph \beta$ sets; for if it had, then the corresponding numbers $\gamma$ would form a sequence of type less than $\omega_{\beta}$, consisting of ordinals less than $\omega_{\beta}$, and with limit $\omega_{\beta}$. Thus $\mu(\beta+1)=\beta+1$. However, $E$ is of cardinality $\kappa \beta$, so that

$$
\mu \infty \leqq \beta+1=\mu(\beta+1) \leqq \mu \infty,
$$

from which it follows that $\mu \alpha=\beta+1$ for all $\alpha$ greater than $\beta$.

Theorem 9 is thus proved. 
Proof of Theorem 7. We now construct a space $E$ by forming the discrete union of spaces of ordinals as in Theorem 9, one for each $\beta$ with $f(\beta+1)=\beta+1$. Let these $\beta$ be $\beta_{1}<\beta_{2}<\ldots<\beta_{k}$, and let the corresponding spaces of ordinals be $E_{i}(1 \leqq i \leqq k)$, with $E=\bigcup\left\{E_{i}: 1 \leqq i \leqq k\right\}$. Now, for each $\alpha$, we have $\mu_{E_{i}} \alpha=0$ if $\alpha \leqq \beta_{i}$, and $\mu_{E_{i}} \alpha=\beta_{i}+1$ if $\beta_{i}+1 \leqq \alpha$, and so $\mu_{E_{i}} \alpha \leqq f \alpha$, since, if $\beta_{i}+1 \leqq \alpha$, then $\beta_{i}+1=f\left(\beta_{i}+1\right) \leqq f \alpha$. Thus $\mu_{E} \alpha \leqq f \alpha$ for all $\alpha$.

On the other hand, $f$ takes only the values 0 or $f\left(\beta_{i}+1\right)$. For there is either no $\beta_{i}$ satisfying $\beta_{i}<\alpha$, or there is a greatest such, say $\beta_{j}$, since there are only finitely many $\beta_{i}$ in all. If there is no such $\beta_{i}$, then clearly $f \alpha=0$. Otherwise, $\left\{\gamma: \beta_{j}<\gamma<\alpha\right\}$ contains no $\beta_{i}$, and so, for every $\gamma$ in this set, $f(\gamma+1)=f \gamma$. Since $f$ is continuous, it follows that $f \gamma=f\left(\beta_{j}+1\right)$ for every $\gamma$, and hence that $f \alpha=f\left(\beta_{j}+1\right)$. Thus, for every $\alpha$, either $f \alpha=0=\mu_{E} \alpha$ or there is a $j$ with $f \alpha=$ $f\left(\beta_{j}+1\right)=\beta_{j}+1$. However, the function $f$ clearly satisfies $f \alpha \leqq \alpha$ for all $\alpha$, so that $\beta_{j}+1 \leqq \alpha$, and so, by Theorem $9, \mu_{E} \alpha=\beta_{j}+1$. So, for each $\alpha$, either $f \alpha \leqq \mu_{E} \alpha$ or there is a $j$ with $f \alpha=\mu_{E} \alpha \leqq \mu_{E} \alpha$. This completes the proof that $\mu_{E}=f$.

The space $E$ is completely regular since each $E_{i}$ is a subspace of a compact Hausdorff space. Thus Theorem 7 is proved.

Theorem 7 gives a complete account of the ordinal functions that can occur as $\mu_{E}$ for spaces $E$ of cardinality less than $\aleph \omega_{0}$. For larger spaces, there are more restrictions on $\mu$.

THEOREM 10. If $\mu 1=0$, then $\mu\left(\omega_{0}+1\right) \leqq \omega_{0}$.

Proof. Let $\Phi$ be a cover of $E$ by exactly $\aleph \omega_{0}$ open sets. Then, as $\aleph \omega_{0}=\aleph 0+\aleph 1+\aleph 2+\ldots$, the cover $\Phi$ can be divided into the disjoint union of subsets $\Phi_{i}$ of cardinality $N i$ (for $\left.i<\omega_{0}\right)$. Then $\left\{U \Phi_{i}: i<\omega_{0}\right\}$ is a cover of $E$ by $\aleph_{0}$ open sets, which, since $\mu 1=0$, has finite subcover, corresponding to the set $N$ of indices, say. But then $\bigcup\left\{\Phi_{i}: i \in N\right\}$ is a subcover of $\Phi$ of cardinality $\aleph \max N$, which is certainly less than $\aleph \omega_{0}$.

THEOREM 11. Let $f$ be an ordinal function satisfying the following conditions:

(i) $f$ is continuous,

(ii) $f 0=0$,

(iii) for each $\alpha, f(\alpha+1)$ is either $\alpha+1$ or $f \alpha$,

(iv) if $f 1=0$, then $f\left(\omega_{0}+1\right) \leqq \omega_{0}$,

(v) $f \infty \leqq \omega_{0}+1$,

(vi) if $f \infty=\omega_{0}+1$, then $f \omega_{0}=\omega_{0}$.

Then there is a completely regular space $E$ with $\mu_{E}=f$.

Proof. It follows at once from (i) and (iii) that $f \alpha \leqq \alpha$ for all $\alpha$, and that $f$ is (non-strictly) increasing. Condition (v) allows three possibilities: $f \infty<\omega_{0}$, or $f \infty=\omega_{0}$, or $f \infty=\omega_{0}+1$. If $f \infty<\omega_{0}$, then Theorem 7 provides what is required.

If $f \infty=\omega_{0}$, suppose that $\gamma$ is the least ordinal with $f \gamma=\omega_{0}$. Then condition (iii) implies that $\gamma$ cannot be a successor. We consider intervals of the form $\{\alpha: \delta<\alpha<\gamma\}$. If $f$ were constant on such an interval, the constant value would be less than $\omega_{0}$, and then, since $f$ is continuous, we should have $f \gamma<\omega_{0}$. Thus there is no such interval on which $f$ is constant; and so, by condition (iii), every such interval contains some $\alpha$ with $f(\alpha+1)=\alpha+1$. Hence 
$\lim _{\alpha<\gamma} f \alpha=\gamma$. But this limit is $f \gamma$; so in fact $\gamma=\omega_{0}$. Thus in this case $f \omega_{0}=\omega_{0}$; and then, as $f$ is continuous at $\omega_{0}$, there must be infinitely many values of $\alpha$ less than $\omega_{0}$ with $f(\alpha+1)=\alpha+1$. Moreover, condition (iv) is vacuously fulfilled, and so imposes no restriction on $f 1$. So we have to consider functions $f$, with $f 0=0, f(\alpha+1)=\alpha+1$ when $\alpha$ belongs to an infinite sequence $\left(\beta_{l}\right)$ of ordinals with $0 \leqq \beta_{1}<\beta_{2}<\ldots<\omega_{0}, f(\alpha+1)=f \alpha$ for all other finite ordinals, and $f \alpha=\omega_{0}$ for all $\alpha$ from $\omega_{0}$ onwards. We construct the appropriate space $E$ in Lemma 1 below.

If, on the other hand, $f \infty=\omega_{0}+1$, and if $\gamma$ is the least ordinal with $f \gamma=\omega_{0}+1$, then, for every $\alpha<\gamma$ we have $f \alpha \leqq \omega_{0}$, so that, as $f$ is continuous, $\gamma$ cannot be a limit. Thus $\gamma$ is a successor, and condition (iii) gives $\gamma=\omega_{0}+1$. So $\omega_{0}<\omega_{0}+1=f\left(\omega_{0}+1\right)$, and condition (iv) now implies that $f 1=1$. Condition (vi) gives also $f \omega_{0}=\omega_{0}$; and hence, as before, there are infinitely many $\alpha$ less than $\omega_{0}$ with $f(\alpha+1)=\alpha+1$. We have then to consider functions $f$ with $f 0=0$, $f(\alpha+1)=\alpha+1$ when $\alpha$ belongs to an infinite sequence $\left(\beta_{i}\right)$ with $0=\beta_{1}<\beta_{2}<\ldots<\omega_{0}$, $f(\alpha+1)=f \alpha$ for all other finite ordinals, $f \omega_{0}=\omega_{0}$ and $f \alpha=\omega_{0}+1$ whenever $\omega_{0}+1 \leqq \alpha$. We construct a space $E$ with such an $f$ as its function $\mu_{E}$ in Lemma 2 below.

Lemma 1. Let $\left(\beta_{i}\right)$ be an infinite sequence of ordinals with $0 \leqq \beta_{1}<\beta_{2}<\ldots<\omega_{0}$. Let the space $X$ be the disjoint union of its subspaces $S, X_{1}, X_{2}, \ldots$, where each $X_{i}$ is homeomorphic to the space of all ordinals less than $\omega_{\beta_{i}}$, with order-topology, and $S$ is a singleton $\{s\}$; and let the topology of $X$ be specified by the requirement that $G$ is open in $X$ if and only if

either $s \notin G$ and, for each $i, G \cap X_{i}$ is open in $X_{i}$,

or $s \in G$ and $G \cap X_{i}$ is open in $X_{i}$ for each $i$, and $X_{i} \subset G$ for nearly all $i$.

Then

$\mu_{X} 0=0$,

$\mu_{X}\left(\beta_{i}+1\right)=\beta_{i}+1$ for each $i$,

$\mu_{X}(\alpha+1)=\mu_{X} \alpha$ for all finite $\alpha$ except the $\beta_{i}$,

and

$\mu_{X} \alpha=\omega_{0}$ for all $\alpha$ from $\omega_{0}$ onwards.

Proof. For each $i$, the space $X$ is the discrete union of its two subspaces $X_{i}$ and $S \cup \bigcup\left\{X_{j}: j \neq i\right\}$. Then Theorems 8 and 9 imply that $\mu_{X_{1}} \leqq \mu_{X}$, and so $\beta_{i}+1=\mu_{X_{i}}\left(\beta_{i}+1\right) \leqq$ $\mu_{X}\left(\beta_{i}+1\right)$. Hence also $\omega_{0} \leqq \mu_{X} \omega_{0}$.

Now let $\Phi$ be any open cover of $X$. Then $s \in G$ for some $G \in \Phi$, and so there is an $i_{0}$ such that $X_{i} \subset G$ whenever $i_{0}<i$. Then $\Phi$ clearly has a subcover comprising at most $\aleph \beta_{i_{0}}$ members. Thus every open cover of $X$ has a subcover by fewer than $N \omega_{0}$ members, so that $\mu_{X} \infty \leqq \omega_{0} \leqq$ $\mu_{X} \omega_{0} \leqq \mu_{X} \infty$. Hence $\mu_{X} \alpha=\omega_{0}$ whenever $\omega_{0} \leqq \alpha$.

We now assume, further, that $\Phi$ is of cardinality less than $\aleph \alpha$, where $\alpha \leqq \beta_{j}$. Then, by Theorem 9 , each subspace $X_{i}$ for $j \leqq i \leqq i_{0}$ is covered by finitely many members of $\Phi$. Hence there is a subcover of $\Phi$ for $X$ of cardinality at most the sum of a finite number and $\aleph \beta_{1}+\aleph \beta_{2}+\ldots+\aleph \beta_{j-1}$. If $2 \leqq j$ this subcover has cardinality $\aleph \beta_{j-1}$. If $j=1$ the sum of cardinals just written down is empty and the subcover is finite. Hence, if $\alpha<\beta_{1}$, then $\mu_{X} \alpha=0$; if $\beta_{i}<\alpha<\beta_{i+1}$, then $\mu_{X} \alpha \leqq \mu_{X}(\alpha+1) \leqq \beta_{i}+1$, and $\mu_{X}\left(\beta_{i}+1\right) \leqq \mu_{X}\left(\beta_{i+1}\right) \leqq \beta_{i}+1$.

Thus, as we have already shown that $\beta_{i}+1 \leqq \mu_{X}\left(\beta_{i}+1\right)$, we have $\mu_{X}\left(\beta_{i}+1\right)=\beta_{i}+1$, and 
hence, if $\beta_{i}<\alpha<\beta_{i+1}$, then $\mu_{X} \alpha \leqq \mu_{X}(\alpha+1) \leqq \beta_{i}+1=\mu_{X}\left(\beta_{i}+1\right)$. But, as $\mu_{X}$ is increasing and as $\beta_{i}+1 \leqq \alpha$, we have also $\mu_{X}\left(\beta_{i}+1\right) \leqq \mu_{X} \alpha$. Thus $\mu_{X}(\alpha+1)=\mu_{X} \alpha$ whenever $\alpha$ is finite but not a $\beta_{i}$.

Finally, it is easy to see that any space constructed as in the statement of the lemma from completely regular spaces $X_{l}$ is itself completely regular.

Lemma 2. Let $0=\beta_{1}<\beta_{2}<\ldots<\omega_{0}$. For each $i$, let $Y_{i}$ be the space of all ordinals less than $\omega_{\beta_{i}}$, and let $Y$ be the discrete union of its subspaces $Y_{i}$ for $i=1,2,3, \ldots$. Then

$\mu_{Y} 0=0$,

$\mu_{Y}\left(\beta_{i}+1\right)=\beta_{i}+1$ for each $i$,

$\mu_{\mathrm{Y}}(\alpha+1)=\mu_{\mathrm{Y}} \alpha$ for all finite $\alpha$ except the $\beta_{i}$,

$\mu_{Y} \omega_{0}=\omega_{0}$,

and

$\mu_{Y} \alpha=\omega_{0}+1$ whenever $\omega_{0}+1 \leqq \alpha$.

Proof. For each $i, Y$ is the discrete union of $Y_{i}$ and $\bigcup\left\{Y_{j}: j \neq i\right\}$, and so Theorems 8 and 9 imply that the values of $\mu_{Y} \alpha$ for finite $\alpha$ are at least those stated. Hence $\mu_{Y} \omega_{0}=\omega_{0}$. The cover of $Y$ by all initial segments of all $Y_{i}$ has no subcover of smaller cardinality, and so $\mu_{Y}\left(\omega_{0}+1\right)=\omega_{0}+1$. As $Y$ is of cardinality $N \omega_{0}$, we have also $\mu_{Y} \infty=\omega_{0}+1$.

Now let $\Phi$ be a covering for $Y$ by fewer than $\aleph \alpha$ open sets, where $\alpha \leqq \beta_{j}$. Then, whenever $j \leqq i$, the subspace $Y_{i}$ is covered by finitely many members of $\Phi$, and so $\Phi$ has a subcover of cardinality at most $\aleph \beta_{1}+\aleph \beta_{2}+\ldots+\aleph \beta_{j-1}+\aleph 0$. If $2 \leqq j$, this number is $\aleph \beta_{j-1}$. On the other hand, if $j=1$ and $\alpha \leqq \beta_{j}$, then $\alpha=0$, and $\mu_{Y} 0=0$. Hence the values of $\mu_{Y} \alpha$ for finite $\alpha$ are at most those stated.

The space $Y$, being a discrete union of completely regular spaces, is itself completely regular.

This completes the proof of Theorem 11. All the hypotheses of this theorem have been shown to be necessary, except conditions (v) and (vi). Whether condition (vi) is necessary remains an open question.

Problem. Does there exist a space $E$ with both the following two properties?

(a) There is a cover of $E$ by $\aleph \omega_{0}$ open sets with no smaller subcover.

(b) For every $n<\omega_{0}$, every cover of $E$ by $\aleph n$ open sets has a countable subcover.

\section{REFERENCES}

1. P. Alexandroff and P. Urysohn, Mémoire sur les espaces topologiques compacts, Nederl. Akad. Wetensch. Proc. Ser. A 14 (1929), 1-96.

2. I. S. Gál, On a generalised notion of compactness I, Nederl. Akad. Wetensch. Proc. Ser. A 60 (1957), 421-430.

3. I. S. Gál, On a generalised notion of compactness II, Nederl. Akad. Wetensch. Proc. Ser. A 60 (1957), 431-435.

4. I. S. Gál, On the theory of $(m, n)$-compact topological spaces, Pacific J. Math. 8 (1958), 721-734.

5. W. Sierpinski, Cardinal and ordinal numbers (Warsaw, 1958).

\section{THE UNIVERSITY}

SHEFFIELD, 10 